\title{
High X-ray-to-optical flux ratio RASS-BSC sources
}

\section{The optical identification}

\author{
L. Cao, J.-Y. Wei, and J.-Y. Hu
}

Beijing Astronomical Observatory, Chinese Academy of Sciences, Beijing 100012, PR China

Received July 27; accepted November 3, 1998

\begin{abstract}
We have used $\log c+0.4 E$ (where $c$ is count rate, and $E$ stands for $E$ magnitude from POSS) as an alternative expression for $\log \left(f_{\mathrm{X}} / f_{\text {opt }}\right)$ to study the $\mathrm{X}$-ray-to-optical flux ratio distribution for known sources in ROSAT All Sky Survey Bright Source Catalog (RASSBSC). Attention focuses on which classes of X-ray emitters have the highest $\mathrm{X}$-ray-to-optical flux ratios. Then a high X-ray-to-optical flux ratio criterion is set and used to select a sample with 34 unidentified X-ray sources from the RASS-BSC for optical spectroscopy. The optical identifications of the sample are presented. Those $34 \mathrm{X}$-ray sources have been identified in the following classes: 10 emission line active galactic nuclei, 7 BL Lacertae objects and 3 BL Lac candidates, 7 clusters of galaxies, 4 Galactic objects and 3 objects remain unidentified due to low signal to noise ratio of the spectra. Identification plausibility is based upon the optical spectroscopy, morphology in the DSS image and the X-ray properties of the sources. We find that preliminary classification can be achieved while extent and hardness ratio in X-ray band, optical morphology and X-ray-to-optical flux ratio are concerned. All of the X-ray sources in the sample are identified with previously known classes of X-ray emitters, and the result is in accord with the $E$ magnitude vs. $(\log c+0.4 E)$ diagram. The proportions of WD and CVs are much higher than that in the EMSS sample. We consider it is due to the lower bandpass of the ROSAT PSPC and the softness of the energy spectrum of these objects. AGN detected in this sample have extremely soft X-ray emission, which infers the presence of a soft X-ray excess in the AGN of this sample. The optical identification results also prove that to find BL Lacertae objects by high X-ray-to-optical flux ratio is an efficient way.
\end{abstract}

Key words: X-rays: galaxies — galaxies: active BL Lacertae objects: general — surveys

Send offprint requests to: L. Cao

\section{Introduction}

The RASS-BSC contains 18811 X-ray sources (Voges et al. $1996 \mathrm{a})$ with a count rate above $0.05 \mathrm{cts} \mathrm{s}^{-1}$. These sources have a detection likelihood of $\geq 15$ and contain at least 15 source photons. Of RASS sources about 65 percent still remain unidentified (Voges et al. 1996b). Several programs have been carried out to identify RASS sources with particular properties.

Previous optical identifications of X-ray sources detected with the Einstein Observatory (EMSS, Stocke et al. 1991) and the EXOSAT satellite (HGLS, Giommi et al. 1991) have shown that different classes X-ray sources occupy apparently different X-ray-to-optical flux ratio range, and those with the highest X-ray-to-optical flux ratio are all spectacular objects such as: BL Lacertae objects, clusters of galaxies, white dwarfs and cataclysmic variable stars etc. Nass et al. (1996) also pointed out that $\log \left(f_{\mathrm{X}} / f_{\mathrm{B}}\right)>1.3$ can be used as an efficient, radio-independent means for selecting candidate BL Lacertae objects while carrying out their RASS sources identification program.

Although to identify high X-ray-to-optical flux ratio sources should have been fruitful, a flux ratio criterion was seldom used at any preselection procedures. The reason for this situation is that there are always several optical objects inside the error circle for an X-ray source, and the flux ratio for an X-ray source will not be the same if a different counterpart is assumed. It will take much time to calculate X-ray and optical flux for each possible counterpart of RASS-BSC Sources if we use a high flux ratio criterion. To make preselection much easier, we make use of the fact that despite Galactic absorbing material $\left(N_{\mathrm{H}}\right) \mathrm{X}$-ray count rate is roughly proportional to X-ray flux. So count rate can replace X-ray flux in the X-rayto-optical flux ratio criterion. For each object inside the X-ray source's error circle, we evaluate its $E$ magnitude and check whether it satisfies the flux ratio criterion. Only those that have at least one object inside the error circle which can fulfil the criterion are included in the sample. 
The paper is ordered as follows: Sect. 2 describes how the alternative flux ratio criterion is constructed and how the sample is defined; Sect. 3 gives an overview on the observation and the data reduction, then optical identification procedure is discussed in detail; in Sect. 4, we report the identification results to the sample and discuss the implication of the results. Final conclusion is given in Sect. 5 .

\section{Flux ratio criterion construction and the sample}

\subsection{Criterion construction}

The alternative flux ratio constraint stems from the statistics to known X-ray sources. Here known sources are the correlation results between RASS-BSC and several catalogs of known objects available in computer-readable format, including catalogs of AGN (Véron-Cetty \& Véron 1996), white dwarfs and NGC, the Bright Star Catalog, $\mathrm{NED}^{1}$ and SIMBAD.

To evaluate X-ray flux for every source in RASSBSC will cost much time. $B$ magnitude of counterparts is not available either ${ }^{2}$. We establish restrict on X-ray count-rate to $E$ magnitude to do preselection from RASSBSC instead, where $E$-magnitude can be derived from the Digitized Sky Survey (DSS) image (Cao et al. 1997). We should point out that not taking Galactic absorbing material into account will reduce precision of the flux ratio constraint and then the efficiency of this preselection method.

We all know that the relationship between magnitude $(m)$ and flux $(f)$ is:

$m=-2.5 \log f+$ constant.

For $E$ magnitude, the relation can be written as:

$E=-2.5 \log f_{\mathrm{E}}+$ constant.

Despite Galactic absorption material $\left(N_{\mathrm{H}}\right)$ count rate $(c)$ is roughly proportional to X-ray flux $\left(f_{\mathrm{X}}\right)$, so with Eq. (2):

$\log \left(f_{\mathrm{X}} / f_{\mathrm{E}}\right)=\log f_{\mathrm{X}}-\log f_{\mathrm{E}} \propto \log c+0.4 E+$ constant.

We can see that for fixed $\log \left(f_{\mathrm{X}} / f_{\mathrm{E}}\right), \log c+0.4 E=$ constant.

$B$ or $V$ magnitudes of known $\mathrm{X}$-ray sources can be drawn from those catalogs that we have mentioned. They must be changed to $E$ magnitude at first. A relation was derived by Humphreys et al. (1991) between the Johnson $B V R$ photometry and the $E$ photographic magnitude on the Palomar plates:

$E-R=-0.011+0.148(V-R)+0.058(V-R)^{4}$

\footnotetext{
1 The NASA/IPAC extragalactic database (NED) is operated by the Jet Propulsion Laboratory, California Institute of Technology, under contract with the National Aeronautics and Space Administration.

${ }^{2}$ Now $B$ and $R$ magnitude are available from USNO-A1.0 (1996, CD-ROM version, U.S. Naval Observatory).
}

for $-0.2<V-R<1.7$

then

$$
E-V=-0.011-0.852(V-R)+0.058(V-R)^{4}
$$

for $-0.2<V-R<1.7$.

Typical color indexes, $(V-R)$ and $(B-V)$, for various types of objects can be drawn from literatures. Now for a known X-ray source, its count rate and $E$ magnitude are available. A plot of $\log c+0.4 E$, versus apparent optical magnitude, $E$ magnitude, is shown in Fig. 1. Only O-M spectral type stars, white dwarfs, emission line AGN and BL Lacertae objects are shown in this graph.

There is an apparent gap between Galactic stars and extragalactic objects except white dwarfs. Emission line AGN concentrate on the discrete area between line A $(\log c+0.4 E=4.9)$ and line $B(\log c+0.4 E=6.4)$. It looks like that BL Lacertae objects have been scatted between $E=14^{\mathrm{m}}$ and $E=20^{\mathrm{m}}$ randomly. Nearly half number of BL Lacertae objects (45 to 105) located at the region above line $\mathrm{B}$, however emission line AGN in this region are only about $3.6 \%$ of its total amount included in the statistic. So taking $\log c+0.4 E \geq 6.4$ as high X-rayto-optical flux ratio criteria can avoid too much normal emission line AGN being included in the sample.

\subsection{The sample}

The high X-ray-to-optical flux ratio sample is defined by following criteria:

1. Exclude known objects. The known objects mean the same as that in Sect. 2.1.

2. Located in the northern hemisphere. In practice we choose $\delta \geq 3^{\circ}$ (epoch 1950), because we can evaluate $E$ magnitude from DSS images only for those objects with declination larger than $3^{\circ}$.

3. High Galactic latitude $\left(|b| \geq 20^{\circ}\right)$, in order to keep away from low Galactic sky area where mainly stars are located.

4. There are counterparts within the circle with radius $D=D_{1}+5^{\prime \prime}$, where $D_{1}$ is the ROSAT X-ray position error given in the RASS-BSC.

5. The $E$ magnitude of the counterpart is less than $18.0^{\mathrm{m}}$, because it is hard to get high enough signal to noise ratio spectrum with a $2 \mathrm{~m}$ telescope for objects fainter than that limit, and meets the high X-ray-to-optical ratio criterion:

$\log c+0.4 E \geq 6.4$.

Thirty-four X-ray sources with Right Ascension between $23^{\mathrm{h}}$ and $13^{\mathrm{h}}$ were selected from RASS-BSC. Table 1 lists the X-ray information for the X-ray sources. There are several cases which have two or more counterparts in the cross-circle. Only those satisfying the above criteria are listed in the Table $1^{3}$. Objects are identified by

\footnotetext{
${ }^{3}$ One exception is 1 RXS J085930.1+745510, its final counterpart is proved not to be the proposed one. We have listed information for the real counterpart too.
} 


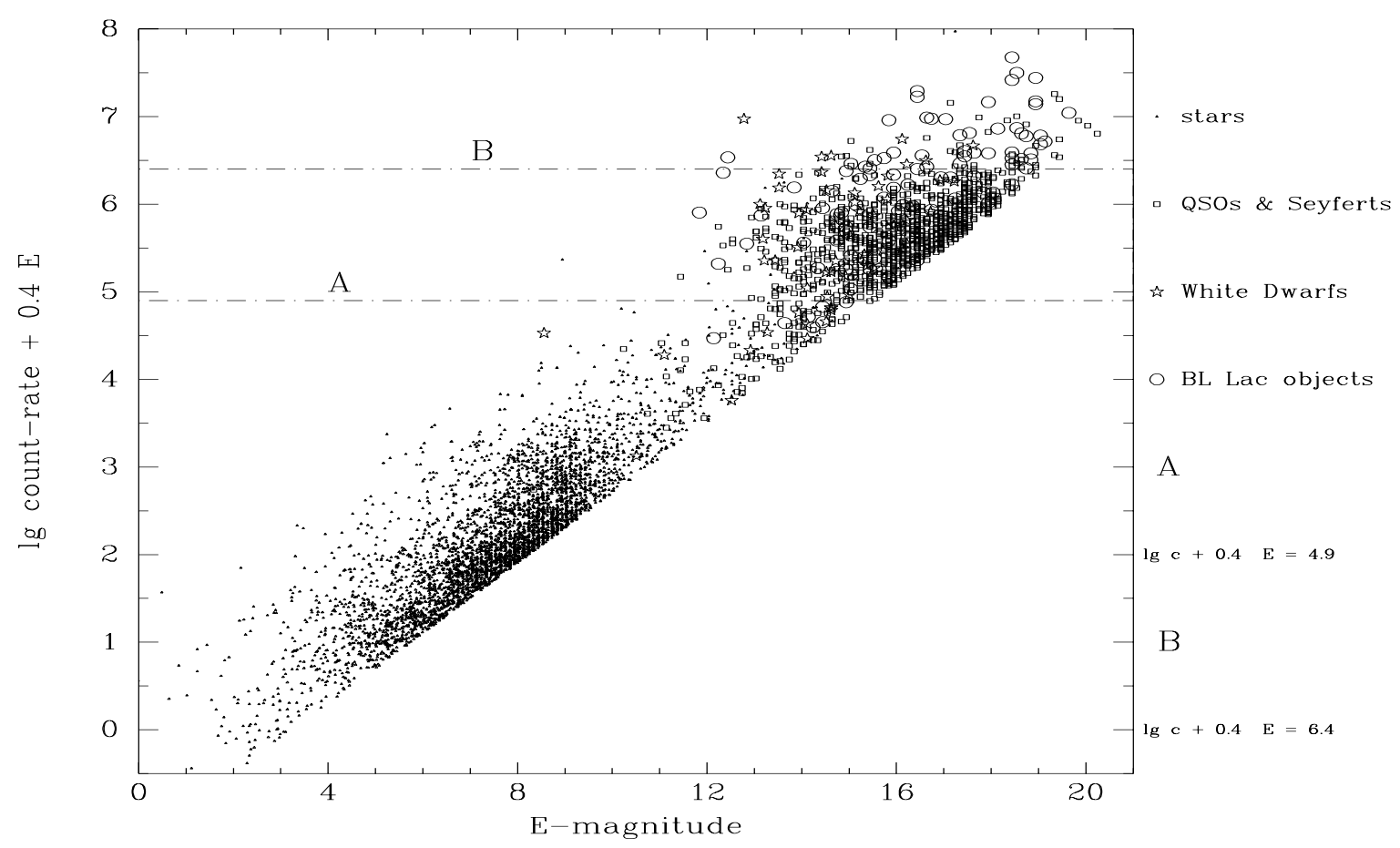

Fig. 1. Plot of $\log c+0.4 E$ vs. $E$-magnitude for known sources

ROSAT name (Col. 1). The optical position (epoch 2000.0, Col. 2 and Col. 3) are derived from the DSS image and should be accurate better than $2^{\prime \prime}$. The X-ray information (e.g. position error, count-rate, hardness 1, and extent in arcsecond) is given in Col. 4 to Col. 7 respectively. These parameters are all given in RASS-BSC and useful for identification procedure. The $E$ magnitude, derived from the DSS images, is given in Col. 8.

\section{Optical identification procedure}

\subsection{The optical spectroscopy and data reduction}

The low resolution spectra of objects in the sample were taken with the $2.16 \mathrm{~m}$ telescope at Xinglong station of Beijing Astronomical Observatory from Dec. 1996 to Mar. 1998. We used OMR spectrograph at the cassegrain focus and a TEK 1024 CCD camera as detector. Two gratings of $200 \AA / \mathrm{mm}$ and $400 \AA / \mathrm{mm}$ were employed in order to get large specral coverage $(>4800 \AA)$, which is broad enough to span all of the gaps between the bright emission-line pairs common in AGN e.g., $\mathrm{Mg}$ II and $\mathrm{H}_{\gamma}$ or $\mathrm{C}$ III] and $\mathrm{Mg}$ II, and to cover identical features for normal galaxies.

He-Ar comparison lamp was used to get wavelength calibration. One to three KPNO standard stars (Massey \& Strobel 1988) were observed to flux calibrate the spectra at every observing night. Because of changing weather conditions, an absolute flux calibration could not be achieved.
All of the data were reduced with standard IRAF procedures. For a few objects, observations were performed twice, and two spectra were combined in order to get a higher $\mathrm{S} / \mathrm{N}$ ratio spectrum. The spectra are shown in Fig. 2 with ROSAT name as labels.

\subsection{Spectroscopic and imaging criteria for classification}

We classify the optical counterparts as Galactic stars or extragalactic sources by means of whether spectral features present redshifts. Only four objects are Galactic stars. Two are white dwarfs with very broad Balmer series in absorption, and a very blue continuum. The other two are cataclysmic variable stars. Their blue nonthermal continuum are distinct. Balmer series emission lines are present in the two spectra.

Extragalactic sources in this sample are classified with emission line AGN, clusters of galaxies, BL Lacterae objects and their candidates. If an extragalactic object possesses broad $\left(F W H M>1000 \mathrm{~km} \mathrm{~s}^{-1}\right)$ and strong $\left(W_{\lambda} \gg\right.$ $5 \AA$ ) emission lines, we classify it as an AGN. BL Lac objects are those with featureless spectra: (i) any emission line that is present, must have $W_{\lambda}<5 \AA$; (ii) if a Ca II "break" is present due to starlight in the BL Lac object host galaxy, it must have a "contrast" $\leq 25 \%$ (Morris et al. 1991). Those with spectrum of a normal galaxy are classified either as clusters of galaxies if a clustering morphology presents in the DSS image, or as BL Lac object 

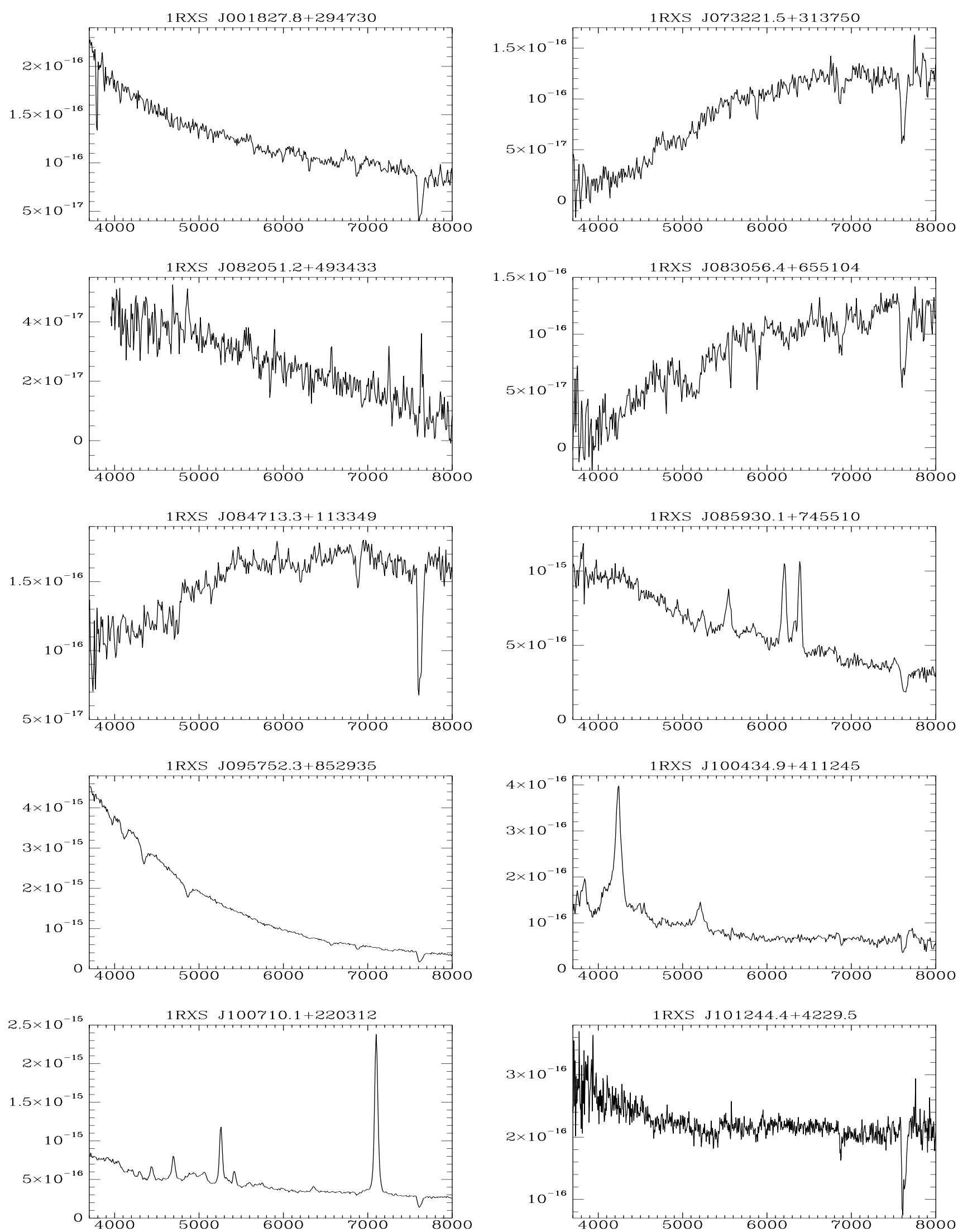

Fig. 2. Optical spectra of the counterparts to the X-ray sources. $f_{\lambda}$ in units of $10^{-15} \mathrm{erg} \mathrm{cm}^{-2} \mathrm{~s}^{-1} \AA^{-1}$ is plotted against wavelength in $\AA$ 

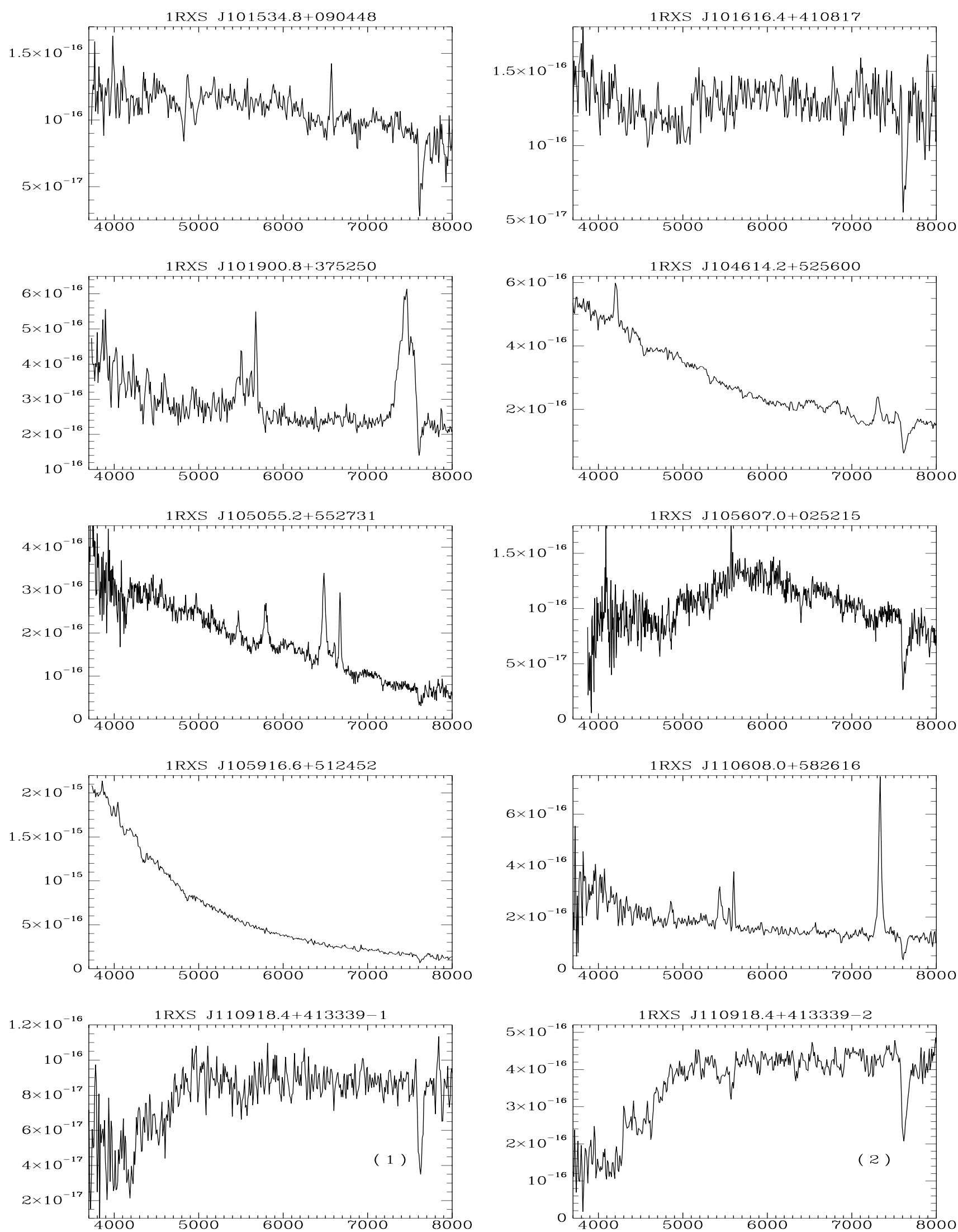

Fig. 2. continued 

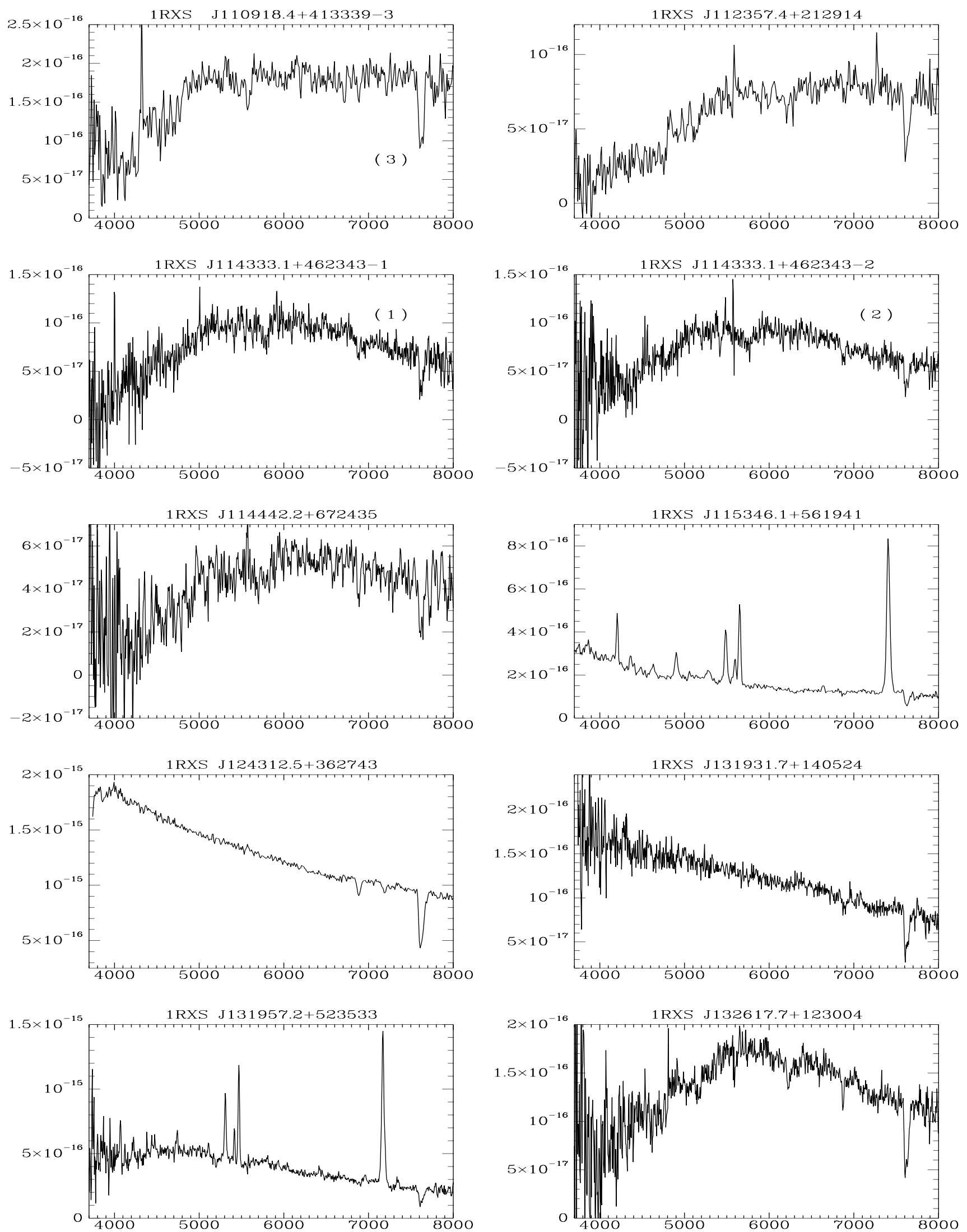

Fig. 2. continued 

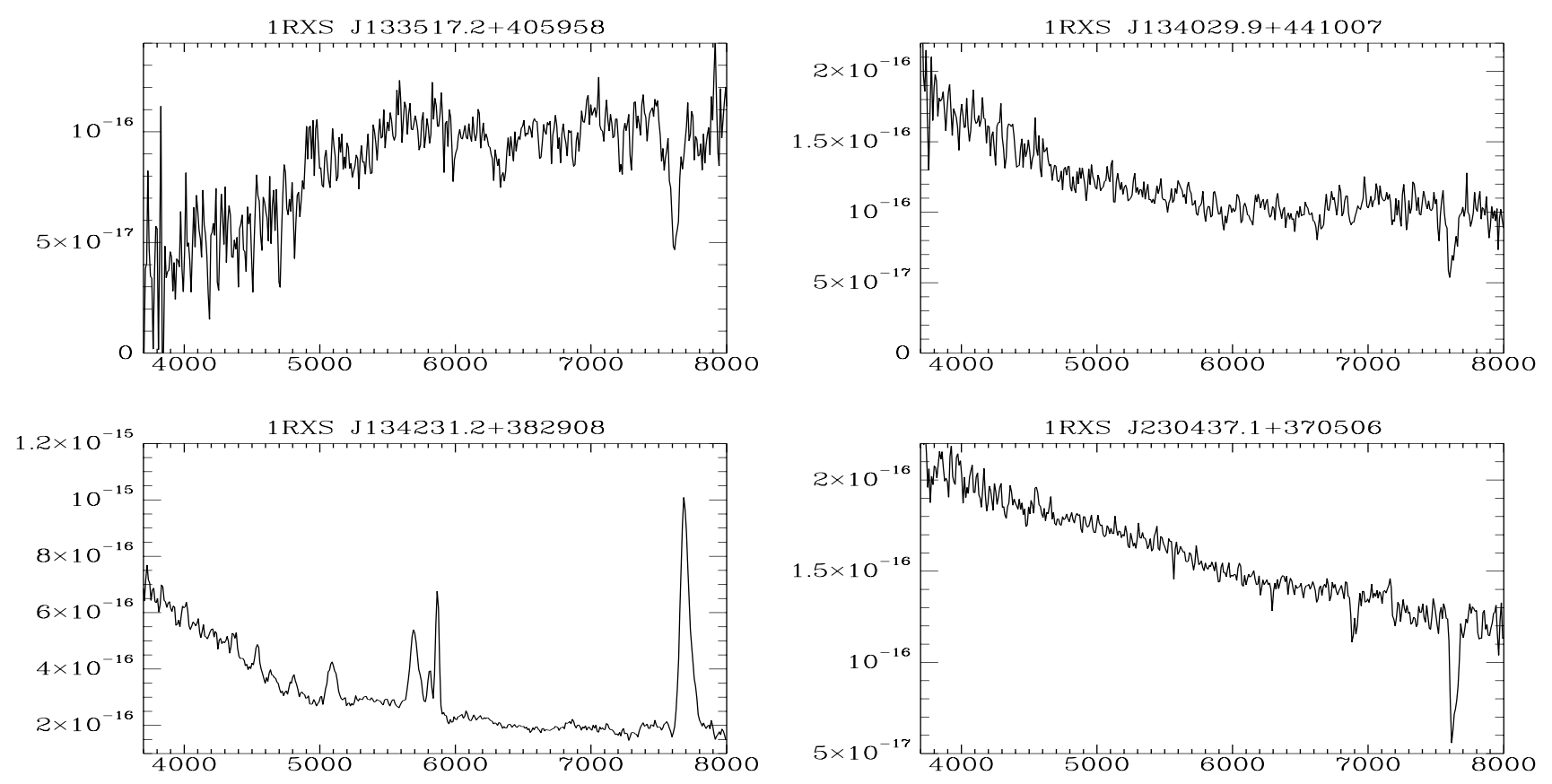

Fig. 2. continued

candidates (The reason for classifying them as BL Lac object candidates but not normal galaxies are illustrated in Sect. 4.4.) if no more objects are located inside or just outside the error circle.

\subsection{Plausibility of identification based on $X$-ray information}

RASS-BSC contains information on X-ray extent, extent likelihood, hardness ratio and other parameters besides count-rate. We use this information to determine whether the proposed optical counterpart to the $\mathrm{X}$-ray source is a plausible one. The X-ray properties of WDs (very soft X-ray colours) and nearby galaxy clusters (hard and extended X-ray emission) have already been noted and used to determine the most plausible counterpart to an X-ray source by Bade et al. (1994, 1995).

According to Fig. 1, Emission line AGN, BL Lac objects and white dwarfs are suitable optical counterparts for objects in this sample. Clusters of galaxies and X-ray binaries are also X-ray luminous objects according to Bade et al. (1994). Normal galaxies are not plausible candidates for this sample's counterparts.

$\mathrm{X}$-ray extent given in RASS-BSC can give a rough view on the size of X-ray emitter. We can use it to determine whether the X-ray source is an extent source or a pointlike one. If we use a conservative extent criterium (extentlikelihood $>10$ and extent $>30$ ) (W. Pietsch et al. 1998) seven sources in the sample are X-ray extended. There are also celestial object groups appearing at the DSS images of these 7 X-ray extended sources. They are all classified as clusters of galaxies by us. Other sources in this sample are neither extent at X-ray band nor in group in optical images.

Two hardness ratios, $H R 1$ and $H R 2$, and their errors are defined in the RASS-BSC:

$$
\begin{aligned}
& H R 1=\frac{H-S}{H+S} \\
& H R 2=\frac{H 1-H 2}{H 1+H 2} .
\end{aligned}
$$

Where $H, S, H 1$ and $H 2$ are the count-rate in the hard $H$-band $(0.5-2.0 \mathrm{keV})$, the soft S-band $(0.1-0.4 \mathrm{keV})$, the hard $H 1$-band $(0.5-0.9 \mathrm{keV})$ and the hard $H 2$-band $(0.9-2.0 \mathrm{keV})$ respectively. Because $H$ is the sum of $H 1$ and $H 2$, and error for $H R 2$ is much higher than for $H R 1$, we only make comparision on $H R 1$ among X-ray sources. In Fig. 3 we give the $H R 1$ distribution of BL Lac objects, Seyferts, QSOs and WDs from which understandings on the hardness ratio for these four classes objects can be constructed. The $H R 1$ for WDs is extremely concentrated between -0.9 and -1.0 . On the contrary, BL Lac objects' $\mathrm{X}$-ray emission is very hard, they seldom occupy the range where $H R 1$ is lower than -0.5 . Of 75 BL Lacertae objects only 2 have $H R 1$ value smaller than -0.5 . In general, AGN don't have very soft X-ray colour.

The spectral classification results are in accord with the statistics. Still, there are some points that are of consideration. In this sample emission line AGN are generally soft $(H R 1<0)$. This situation may have connection with their higher X-ray-to-optical flux ratio. We have no statistical information on the $H R 1$ distribution for $\mathrm{CVs}$ and clusters of galaxies, but clusters of galaxies are generally 

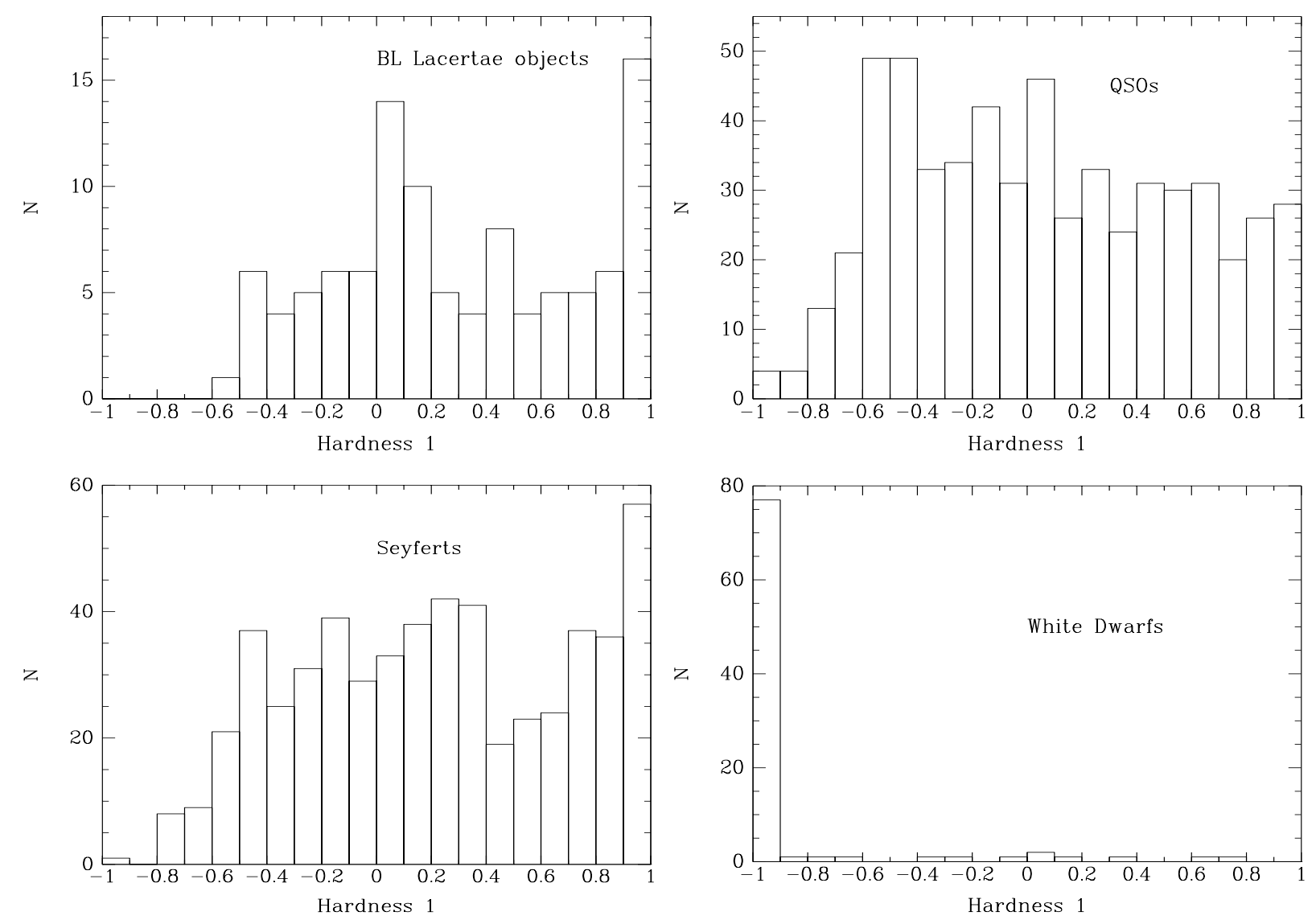

Fig. 3. Hardness 1 for BL Lacertae Objects, QSOs, Seyferts and White Dwarfs

accepted as possessing hard X-ray colour. Both CVs in this sample have $H R 1$ smaller than -0.8 , but it may be not the common sense for CVs. According to Richman's (1996) research, only about half number of CVs have soft X-ray excess.

\section{Results and discussion}

\subsection{Identification results}

Out of 34 X-ray sources, thirty-one have been fully identified. The other three proposed optical counterparts to the X-ray sources are too faint to recognize any features from their spectra or even to do spectrometry with a $2 \mathrm{~m}$ telescope. The optical counterparts are all the proposed objects except 1RXS J085930.1+745510 whose counterpart is a bright $\left(E=15.8^{\mathrm{m}}\right)$ AGN located southwest the proposed one. Final identification results are provided in Table 1. The identification classification of the counterpart based upon the criteria described in Sect. 3 is listed in Col. 10. For most of the extragalactic objects, redshifts are determined and given in Col. 10. Column 11 comments on main features present in the spectrum that give clues to the spectral classification. The distribution of two hardness ratios for objects in the sample is shown in Fig. 4.

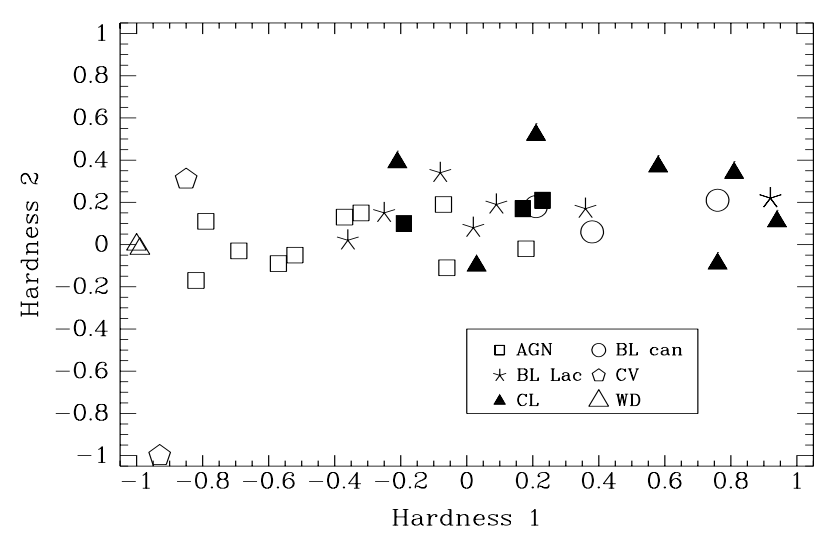

Fig. 4. Hardness ratios for the objects in the sample

\subsection{Selection efficiency for BL Lacertae objects}

BL lacertae objects are rare AGN which are characterized by "featureless" spectra, highly variable, and strong polarization in the radio to optical emission. Its peculiar spectral characteristic prevents it from being discovered in optical domain (Green et al. 1986; Flemming et al. 1991). At first, BL Lacertae objects have been discovered almost by their radio emission (Kühr et al. 1990; 
Table 1. X-ray identification information

\begin{tabular}{|c|c|c|c|c|c|c|c|c|c|c|}
\hline $\begin{array}{l}\text { ROSAT name } \\
\text { 1RXS J } \\
(1)\end{array}$ & $\begin{array}{l}\text { R.A. } \\
(\mathrm{h} \mathrm{m} \mathrm{s}) \\
(2)\end{array}$ & $\begin{array}{r}\text { Decl. } \\
(\mathrm{d} \mathrm{m} \mathrm{s}) \\
(3)\end{array}$ & $\begin{array}{r}\text { P.E. } \\
\left({ }^{\prime \prime}\right) \\
(4)\end{array}$ & $\begin{array}{c}C R \\
\operatorname{cts~s}^{-1} \\
(5)\end{array}$ & $H R 1$ & $\begin{array}{l}\text { ext } \\
\left({ }^{\prime \prime}\right) \\
(7)\end{array}$ & $\begin{array}{c}E \\
m \\
(8)\end{array}$ & $\mathrm{ID}^{\dagger}$ & $\begin{array}{c}z \\
(10)\end{array}$ & Comment \\
\hline $001827.8+294730$ & 001827.7 & 294733 & 7 & 0.460 & 0.36 & 7 & 17.3 & BL & & \\
\hline $073221.5+313750$ & 073221.2 & 313745 & 11 & 0.364 & 0.81 & 55 & 17.7 & CL & 0.35 & $\mathrm{H}_{\gamma}, \mathrm{H}_{\delta}$ in absorption \\
\hline $082051.2+493433$ & 082050.9 & 493432 & 9 & 0.249 & -0.93 & 0 & 17.8 & $\mathrm{CV}$ & & EL: $\mathrm{H}_{\alpha}, \mathrm{H}_{\beta}, \mathrm{O}$ I, Fe II \\
\hline $083056.4+655104$ & 083058.8 & 655053 & 15 & 0.569 & 0.94 & 97 & 17.8 & CL & 0.210 & $\mathrm{H}_{\beta}, \mathrm{Mg} \mathrm{b}, \mathrm{Ca}$ II $\mathrm{H} \& \mathrm{~K}$ \\
\hline $084713.3+113349$ & 084712.9 & 113352 & 8 & 0.933 & 0.38 & 11 & 16.9 & BL can & 0.198 & Mg b, Ca II H\&K, " $G$ " band \\
\hline \multirow[t]{2}{*}{$085930.1+745510$} & 085932.4 & 745519 & 8 & 0.163 & 0.18 & 0 & 17.9 & & & \\
\hline & 085930.9 & 745458 & & & & & 15.8 & QSO & 0.276 & EL: H I, [O III] \\
\hline $092401.1+053350$ & 092401.0 & 053346 & 10 & 0.202 & 0.17 & 21 & 17.8 & & & too faint to be observed \\
\hline $095752.3+852935$ & 095754.1 & 852943 & 7 & 0.544 & -1.00 & 15 & 17.0 & WD & & broad H I absorption lines \\
\hline $100434.9+411245$ & 100434.8 & 411243 & 8 & 0.236 & -0.32 & 9 & 17.9 & QSO & 1.734 & EL: C IV, Ciri], Si IV/O IV] \\
\hline $100710.1+220312$ & 100710.2 & 220302 & 8 & 0.621 & -0.06 & 18 & 17.0 & Sey & 0.082 & EL: H I, [O III], He I \\
\hline $101244.4+422959$ & 101244.2 & 422958 & 7 & 0.664 & 0.02 & 14 & 17.1 & BL & 0.364 & Ca II H\&K, "G" band \\
\hline $101534.8+090448$ & 101534.7 & 090445 & 8 & 1.145 & -0.85 & 0 & 16.6 & $\mathrm{CV}$ & & $\mathrm{EL}: \mathrm{H}_{\alpha}, \mathrm{H}_{\beta}$ \\
\hline $101616.4+410817$ & 101616.7 & 410813 & 7 & 0.480 & -0.08 & 7 & 17.3 & $\mathrm{BL}$ & $\sim 0.27$ & Ca II break \\
\hline $101900.8+375250$ & 101900.4 & 375242 & 7 & 0.775 & -0.07 & 5 & 17.2 & Sey & 0.133 & EL: $\mathrm{H}_{\alpha}, \mathrm{H}_{\beta},[\mathrm{O}$ III $]$ \\
\hline $104614.2+525600$ & 104614.2 & 525600 & 8 & 0.322 & -0.82 & 0 & 17.6 & QSO & 0.503 & EL: $\mathrm{Mg}$ II, $\mathrm{H}_{\beta},[\mathrm{O}$ III $]$ \\
\hline $105055.2+552731$ & 105055.1 & 552725 & 7 & 0.374 & -0.69 & 9 & 17.1 & QSO & 0.333 & EL: $\mathrm{H} \mathrm{I},[\mathrm{O}$ III $]$ \\
\hline $105607.0+025215$ & 105606.6 & 025215 & 9 & 0.585 & 0.76 & 19 & 17.2 & BL can & 0.236 & Mg b, Ca II H\&K, " $G$ " band \\
\hline $105916.6+512452$ & 105916.3 & 512444 & 6 & 4.180 & -0.99 & 18 & 17.3 & WD & & broad H I absorption lines \\
\hline $110608.0+582616$ & 110607.8 & 582609 & 8 & 0.269 & -0.79 & 19 & 17.5 & Sey & 0.128 & EL: H I, [O III] \\
\hline $110748.2+150217$ & 110748.2 & 150214 & 8 & 0.453 & -0.19 & 0 & 17.6 & & & $\mathrm{~S} / \mathrm{N}$ too low to be classified \\
\hline $110918.4+413339$ & 110917.8 & 413347 & 20 & 0.250 & 0.76 & 86 & 17.5 & CL & & \\
\hline \multirow[t]{3}{*}{$112357.4+212914$} & 112356.7 & 212911 & 16 & 0.284 & 0.21 & 76 & 17.9 & CL & 0.199 & Mg b, Ca II H\&K, " $G$ " band \\
\hline & 112356.8 & 212923 & & & & & 18.0 & & & \\
\hline & 112356.7 & 212911 & & & & & 17.4 & & & \\
\hline \multirow{2}{*}{$114333.1+462343$} & 114333.0 & 462340 & 48 & 0.388 & 0.58 & 50 & 17.3 & CL & & \\
\hline & 114331.7 & 462327 & & & & & 17.2 & & 0.11 & Mg b, Ca II H\&K, "G" band \\
\hline $114442.2+672435$ & 114441.4 & 672447 & 13 & 0.380 & -0.21 & 82 & 17.9 & CL & & \\
\hline $114930.4+243928$ & 114930.2 & 243928 & 7 & 0.616 & 0.23 & 13 & 17.1 & & & $\mathrm{~S} / \mathrm{N}$ too low to be classified \\
\hline $115346.1+561941$ & 115345.9 & 561936 & 10 & 0.272 & -0.57 & 21 & 17.5 & Sey & 0.129 & EL: H I, [O III] \\
\hline $124312.5+362743$ & 124312.7 & 362746 & 7 & 1.297 & -0.36 & 15 & 16.4 & $\mathrm{BL}$ & & \\
\hline $131931.7+140524$ & 131931.7 & 140534 & 8 & 0.613 & -0.25 & 0 & 17.8 & BL & & \\
\hline $131957.2+523533$ & 131957.0 & 523534 & 7 & 0.663 & -0.52 & 12 & 17.3 & Sey & 0.092 & EL: H I, [O III], [O II], [Ne III] \\
\hline $132617.7+123004$ & 132617.7 & 123000 & 8 & 0.382 & 0.21 & 12 & 17.2 & BL can & 0.204 & Mg b, Ca II H\&K, " $G$ " band \\
\hline $133517.2+405958$ & 133516.8 & 405949 & 14 & 0.388 & 0.03 & 80 & 17.5 & CL & 0.223 & Mg b, Ca II H\&K, " $G$ " band \\
\hline $134029.9+441007$ & 134029.8 & 441004 & 8 & 0.296 & 0.09 & 15 & 17.6 & $\mathrm{BL}$ & & \\
\hline $134231.2+382908$ & 134231.2 & 382905 & 8 & 0.365 & -0.37 & 19 & 17.4 & Sey & 0.172 & EL: H I, [O III], [O II], [Ne III] \\
\hline $230437.1+370506$ & 230436.6 & 370508 & 7 & 0.430 & 0.92 & 17 & 17.5 & BL & & \\
\hline
\end{tabular}

†BL: BL Lacertae object.

CL: Cluster of galaxies.

CV: Cataclysmic variable star.

BL can: BL Lac object candidate.

WD: White dwarf.

Sey: Seyfert.

Stickel et al. 1991; Stickel et al. 1993; Burbidge \& Hewitt 1987). Since last decade the identification of X-ray sources has been proved to be a more efficient way to search for BL Lac objects (EMSS, Stocke et al. 1991; Giommi et al. 1991; Elvis et al. 1992). Because the identification of large $\mathrm{X}$-ray sky survey sample is very time consuming, some kind of pre-selection ought to be made to get a higher BL Lac object fraction. Several teams made use of the fact that BL Lac objects have typical broadband spectral indices in their finding BL Lac objects program (Schachter et al. 1993; Nass et al. 1996). Considerations both on broadband spectral distribution and optical polarimetry lead to fruitful results (Kock et al. 1996).

Seven BL Lacertae objects found in the sample are all newly discovered. The efficiency of the high X-ray-tooptical selection $(>20 \%)$ is higher than for most other techniques (Kock et al. 1996) and much higher than the prediction for the potential of RASS in finding BL Lac 
Table 2. Evaluated X-ray parameters for three normal galaxies ${ }^{\ddagger}$

\begin{tabular}{ccccccccc}
\hline \hline $\begin{array}{c}\text { ROSAT name } \\
\text { 1RXS J }\end{array}$ & $z$ & $C R$ & $\begin{array}{c}D^{\mathrm{L}} \\
\mathrm{Mpc}\end{array}$ & $\begin{array}{c}L_{\mathrm{X}} \\
\mathrm{erg} \mathrm{s}^{-1}\end{array}$ & $\begin{array}{c}D^{\mathrm{A}} \\
\mathrm{Mpc}\end{array}$ & $\begin{array}{c}\text { size } \\
\left({ }^{\prime \prime}\right)\end{array}$ & $\begin{array}{c}\text { ext } \\
\left({ }^{\prime \prime}\right)\end{array}$ & $\begin{array}{c}f_{\mathrm{r}} \\
\mathrm{mJy}\end{array}$ \\
\hline $084713.3+113349$ & 0.198 & 0.9327 & 1242 & $1.7210^{45}$ & 865 & 72 & 11 & 33.2 \\
$105607.0+025215$ & 0.236 & 0.5850 & 1491 & $1.5610^{45}$ & 976 & 63 & 19 & 4.3 \\
$132617.7+123004$ & 0.204 & 0.3822 & 1281 & $7.5010^{44}$ & 884 & 70 & 12 & 58.1 \\
\hline
\end{tabular}

$\ddagger H_{0}=50 \mathrm{~km} \mathrm{~s}^{-1} \mathrm{Mpc}^{-1}$ is assumed.

objects $^{4}$. We have noted that the X-ray information quoted in RASS-BSC can give more constraint on preselection. Most BL Lacertae objects are point sources in $\mathrm{X}$-ray and possess $H R 1$ larger than -0.5 . If these two restrictions had been supplied at the beginning of sample selection, we would have found 7 BL Lac objects out of 18 X-ray sources. The fraction of BL Lacertae objects would have been doubled.

\subsection{Soft $X$-ray excess of emission line AGNs}

ROSAT PSPC worked on a rather soft X-ray band (0.1 $2.4 \mathrm{keV}$ ) compared with previous X-ray missions. This situation provides a probe into the soft X-ray property of X-ray emitters. Among the ten AGN detected in the sample, nine have rather small $H R 1(H R 1<0)$, one exception is 1RXS J085930.1+745510 whose counterpart is not the proposed one. By definition small $H R 1, H R 1<0$, is to say that more than $50 \%$ of the counts are in soft band $(0.1-0.4 \mathrm{keV})$. Generally, a negative $H R 1$ presents a steeper X-ray spectrum than a positive one (Grupe et al. 1997). The steep X-ray spectra can indicate either the presence of a soft X-ray excess over a flat hard X-ray continuum as a part of a Big Blue Bump (BBB) which extends from the UV spectral range to soft X-ray energies, or a systematic lack of emission around $1 \mathrm{keV}$ and above which may be caused by warm absorbers (Boller et al. 1996). If we take the sample selection criterion into consideration, the former case should be followed.

Supposing intrinsic optical to X-ray indices for AGN are the same, lack of emission at hard X-ray caused by warm absorber or other possible mechanic would reduce total emission in X-ray band. As a result, an AGN with steep X-ray spectrum must be less luminous than those with flat spectra in X-ray band. It is obviously opposite to the identification results of our sample because it is a high X-ray-to-optical flux ratio selected one. We conclude that the soft $H R 1$ of AGN in the sample is an indicator of soft X-ray excess.

\footnotetext{
${ }^{4}$ We have not taken BL Lac object candidates into accout, otherwise the selection efficiency would be higher.
}

\subsection{BL Lac object candidates as the counterparts}

Three proposed counterparts of X-ray sources are classified as BL Lac object candidates. Their spectra show marked normal galaxy features such as Ca II break, " $G$ " band etc. We did not take them as normal galaxies because their X-ray luminosity largely exceeds the typical value for elliptical galaxies. We use lower conversion factor between the count rate and flux, $10^{-11} \mathrm{erg} \mathrm{cm}^{-2} \mathrm{~s}^{-1}$, which corresponds to a relatively low galactic foreground absorption, to estimate the lower limit X-ray flux of X-ray sources. For an Einstein-de Sitter universe model $\left(q_{0}=\frac{1}{2}\right)$, the luminosity distance takes the form:

$D^{\mathrm{L}}=\frac{2 c}{H_{0}}[(1+z)-\sqrt{1+z}]$.

The redshift, $D^{\mathrm{L}}$ and estimated X-ray luminosity for the three proposed galaxies are listed in Table 2. The X-ray luminosity of the three objects is largely in excess of the constraint for elliptical galaxies drawn from EMSS (Stocke et al. 1991) even if the difference in the bandpass between Einstein IPC and ROSAT PSPC is concerned. So it is impossible to take normal galaxies as the counterparts of those three X-ray sources.

BL Lacertae objects are very luminous X-ray emitters, a galaxy with an active galactic nucleus can count for such high X-ray luminosity. On the other hand, BL Lacertae object is featureless in optical regime, it can be easily submerged with starlight from the host galaxy. Browne and Marchã have noticed that the possibility of misclassifying $\mathrm{X}$-ray sources as elliptical galaxies or clusters of galaxies is marked (Browne \& Marchã 1993). All of the three sources can also find their entries in the NRAO VLA Sky Survey (Condon et al. 1998). Table 2 gives the radio flux from NVSS for the three sources. So long as these facts are concerned, the probability that the three sources are in fact BL Lac objects is very high.

Approach to find other plausible counterparts is failed since there is no other objects inside or just outside the error circle in the DSS image. However, the probable counterparts to the three X-ray sources may also be,

(a) clusters of galaxies. The proposed counterparts are the major $\mathrm{cD}$ galaxies of the clusters, other cluster members are too faint to present in DSS images. However, redshift around 0.2 is a quite medium 
value for clusters of galaxies, clusters with such a redshift should have presented cluster morphology in DSS (Stocke et al. 1991) and extent X-ray emission.

(b) The proposed optical counterparts are projections by chance, and the real counterparts are too faint to be found in DSS images. Since optical blank field cases are common in X-ray sources identification, projection by chance is an acceptable explanation. The real counterparts to them may be distant clusters of galaxies or BL Lacertae objects.

\subsection{Efficiency in finding $W D$ and $C V s$}

The proportions of WDs and CVs are high in the sample. That is another beneficial result of the softness of ROSAT PSPC bandpass: efficient in finding white dwarfs and cataclysmic variable stars. Most white dwarfs release energy mainly at bandpass below $0.4 \mathrm{keV}$ (see Fig. 3), and half numbers of CVs possess soft X-ray excess according to Richman's (1996) investigation. The peculiar X-ray spectrum of these sources makes them difficult to detect in energy bands harder than that of ROSAT.

\section{Conclusion}

This is a rather small sample compared with 18811 entries in RASS-BSC. However it gives us a view into what kind of objects occupy the highest X-ray-to-optical flux ratio regime. We can draw following conclusion from the sample identification results:

1. Finding BL Lac objects by identifying X-ray sources with very high $\mathrm{X}$-ray-to-optical flux ratio is proved to be fruitful.

2. Those X-ray parameters listed in RASS-BSC can give a clue to the optical counterparts of X-ray sources. Pre-identification can be achieved if the combination properties in X-ray band (count-rate, hardness ratio and extent) and optical band (visual magnitude and morphology) are concerned.

3. The peculiar $H R 1$ distribution of AGN predicts that there is a soft X-ray excess over the flat hard X-ray continuum of AGN in the sample.
Acknowledgements. We thank an anonymous referee for valuable comments and suggestions. Dr. D.-W. Xu, X.-J. Jiang and Dr. Z.-H. Zhu give us much help in preparing the manuscript. This work is partly supported by the Pandeng Project of the Chinese Scientific Committee and the Chinese National Natural Science Foundation.

\section{References}

Bade N., Fink H.H., Engels D., 1994, A\&A 286, 381

Bade N., Fink H.H., Engels D., et al., 1995, A\&AS 110, 469

Boller Th., Brandt W.N., Fink H.H., 1996, A\&A 305, 53

Browne I.W.A., Marchã M.J.M., 1993, MNRAS 261, 795

Burbidge G., Hewitt A., 1987, ApJ 92, 1

Cao L., Wei J.-Y., Hu J.-Y., 1997, Acta Astrophys. Sin. 17, No. 4,428

Condon J.J., Cotton W.D., Greisen E.W., et al., 1998, AJ 115 1693

Flemming T.A., Green R., Jannuzi B., et al., 1993, AJ 106, 1729

Elvis M., Plummer D., Schachter J., Fabbiano G., 1992, ApJS 80,257

Giommi P., Tagliaferri G., Beuermann K., et al., 1991, ApJ 378,77

Green R., Schmidt M., Liebert J., 1986, ApJS 61, 305

Grupe D., Beuermann K., Thomas H.-C., Mannheim K., Fink H.H., 1998, A\&A 330, 25

Humphreys R.M., LanDau R., Ghigo F., Zumach W., Labonte A., 1991, AJ 102, 1

Kock A., Meisenheimer K., Brinkmann W., Neumann M., Siebert J., 1996, A\&A 307, 745

Kühr H., Schmidt G.D., 1990, AJ 99, 1

Massey P., Strobel K., 1988, ApJ 328, 315

Morris S.L., Stocke J.T., Gioia I.M., et al., 1991, ApJ 380, 49

Nass P., Bade N., Kollgaard R.I., et al., 1996, A\&A 309, 419

Pietsch W., Bischoff K., Boller Th., et al., 1998, A\&A 333, 48

Richman H.R., 1996, ApJ 462, 404

Schachter J.F., Stocke J.T., Perlman E., et al., 1993, ApJ 412, 541

Stickel M., Padovani P., Urry C.M., Fried J.W., Kühr H., 1991, ApJ 374, 431

Stickel M., Fried J.W., Kühr H., 1993, A\&AS 98, 393

Stocke J.T., Morris S.L., Gioia I.M., Maccacaro T., Schild R.E., Wolter A., 1990, ApJ 348, 141

Stocke J.T., Morris S.L., Gioia I.M., et al., 1991, ApJS 76, 813

Véron-Cetty M.P., Véron P., 1996, ESO Sci. Rep. No. 17

Voges W., Aschenbach B., Boller T., et al., 1996a, IAU Circ. 6420

Voges W., Boller Th., Dennerl K., et al., 1996b, MPE Report 263,637 